\title{
PPARD rs2016520 (T/C) and NOS1AP rs12742393 (A/C) polymorphisms affect therapeutic efficacy of nateglinide in Chinese patients with type 2 diabetes mellitus
}

\author{
Tao Wang ${ }^{1,2+}{ }^{\text {, Jin-Fang Song }}{ }^{1,3+}$, Xue-Yan Zhou ${ }^{1}$, Cheng-Lin Li ${ }^{1}$, Xiao-Xing Yin ${ }^{1}$ and Qian Lu ${ }^{1 *}$
}

\begin{abstract}
Background: Genetic polymorphisms in the PPARD and NOSIAP is associated with type 2 diabetes mellitus (T2DM); however, there is no evidence about its impact on the therapeutic efficacy of nateglinide. This study was designed to investigate a potential association of PPARD rs2016520 (T/C) and NOS1AP rs12742393 (A/C) polymorphisms with efficacy of nateglinide in newly diagnosed Chinese patients with type 2 diabetes mellitus (T2DM).

Methods: Sixty patients with newly diagnosed T2DM were enrolled to identify PPARD rs2016520 and NOS1AP rs12742393 genotypes using the polymerase chain reaction-restriction fragment length polymorphism assay (PCRRFLP). All subjects were treated with nateglinide (360 mg/day) for 8 weeks. Anthropometric measurements, clinical laboratory tests were obtained at baseline and after 8 weeks of nateglinide treatment.
\end{abstract}

Results: After nateglinide treatment for 8 consecutive weeks, patients with at least one $C$ allele of PPARD rs2016520 showed a smaller decrease in post plasma glucose (PPG), homeostasis model assessment for beta cell function (HOMA-B) than those with the TT genotype did $(P<0.05)$. In patients with the AA genotype of NOS1AP rs12742393, the drug showed better efficacy with respect to levels of fasting plasma glucose (FPG), fasting serum insulin (FINS), HOMA-B and homeostasis model assessment for insulin resistance (HOMA-IR) than in patients with the AC + CC genotype $(P<0.05)$. NOS1AP rs 12742393 genotype distribution and allele frequency were associated with responsiveness of nateglinide treatment $(P<0.05)$.

Conclusions: The PPARD rs 2016520 and NOS1AP rs 12742393 polymorphisms were associated with nateglinide monotherapy efficacy in Chinese patients with newly diagnosed T2DM.

Trial registration: Chinese Clinical Trial Register ChiCTR13003536, date of registration: May 14, 2013.

Keywords: PPARD, NOS1AP, Genetic polymorphism, Type 2 diabetes mellitus, Nateglinide

*Correspondence: prairy@126.com

${ }^{\dagger}$ Tao Wang and Jin-Fang Song have contributed equally to this study.

1 Jiangsu Key Laboratory of New Drug Research and Clinical Pharmacy,

Xuzhou Medical University, Xuzhou, China

Full list of author information is available at the end of the article

\begin{abstract}
Introduction
Nateglinide is an important non-sulfonylurea oral hypoglycemic agent that promotes insulin secretion from pancreatic islet beta cells by inhibiting ATP-sensitive $\mathrm{K}^{+}$ channels and activating $\mathrm{Ca}^{2+}$ channels $[1,2]$. However, considerable interindividual differences in the therapeutic efficacy of nateglinide have been reported in patients with T2DM $[1,2]$. The underlying mechanism is still
\end{abstract}


unclear. It is hypothesized that genetic polymorphisms of genes that code drug metabolizing enzymes, drug transporters, drug targets, or susceptibility genes related to T2DM pathogenesis may affect the pharmacokinetic or pharmacodynamics process of drugs, and eventually lead to interindividual variation in therapeutic efficacy of nateglinide [30]. Cytochrome P450 (CYP) 2C9 and CYP3A4 have been identified as the main metabolic enzymes involved in the biotransformation of nateglinide [3]. SLCO1B1 gene encoding organic anion transporting polypeptide 1B1 (OATP1B1), which is involved in cellular uptake and transport of nateglinide [3]. The interindividual differences may be attributed to genetic polymorphism of $C Y P 2 C 9$ and $S L C O 1 B 1$, but not the CYP3A4 polymorphisms [3]. Nevertheless, this could not elucidate all the causes of various nateglinide responses $[4,5]$.

PPARD is located on chromosome 6p21.1-p21.2, and its coding product PPAR- $\delta$ (also named PPAR- $\beta$ ) is a member of the peroxisome proliferator activated receptor family, which is widely distributed in the liver, kidneys, cardiac and skeletal muscle, adipose tissue, brain, pancreatic and vasculature [6]. PPAR- $\delta$ plays an important role in insulin resistance and islet $\beta$-cell function [7-9]. More recently, PPAR- $\delta$ activation came into focus as an interesting novel approach for the treatment of metabolic syndrome. Meanwhile, both preclinical and clinical studies have shown that PPAR- $\delta$ specific agonist therapy enhanced $\beta$-oxidation, decreased free fatty acid, and improved insulin sensitivity $[10,11]$. Largescale clinical studies in the Chinese population have shown that PPARD rs2016520 polymorphism (also named $+294 \mathrm{~T}>\mathrm{C}$ or $-87 \mathrm{~T}>\mathrm{C}$ ) is associated with blood glucose, insulin level and insulin resistance, and is a key factor affecting the development of metabolic syndrome and T2DM $[12,13]$. Studies in a Mexican population have produced similar results [14].

NOS1AP, located on chromosome 1q22.3, and its coding product is known as carboxy-terminal PDZ ligand of neuronal nitric oxide synthase (nNOS), which regulates nNOS activity through interaction with the PDZ binding region of nNOS [15]. It was shown that elevated levels of $\mathrm{Ca}^{2+}$ in islet $\beta$-cells can activate nNOS in the surface layer of insulin secretory granules and participate in the insulin secretion process [15]. In addition, the administration of cholesterol to inhibit nNOS activity can impede the process of insulin secretion, further suggesting its biological role in insulin secretion [16, 17]. The NOS1AP rs12742393 polymorphism was found to be associated with susceptibility to new-onset diabetes in patients treated with calcium channel blockers in different populations, and pharmacogenomic studies have shown that NOS1AP polymorphisms are one of the important factors contributing to differences in the efficacy of hypoglycemic agents, especially sulfonylureas [18, 19]. One clinical study showed that NOS1AP rs12742393 $\mathrm{C}$ allele gene was associated with an increased susceptibility to T2DM in the Chinese population [20]. Though the studies on how the variants influenced the diseases were limited, one functional study showed that rs12742393 could affect NOS1AP expression through influencing transcription factor binding [21]. To date, there has been no report about the effect of PPARD rs2016520 and NOS1AP rs12742393 polymorphism on nateglinide response. Therefore, the PPARD rs2016520 and NOS1AP rs12742393became our focus.

Based on the facts that PPARD and NOS1AP play crucial roles in functional regulation of $\beta$-cells, insulin resistance and metabolism, we conduct this study to identify the association PPARD rs2016520 (T/C) and NOS1AP rs12742393 (A/C) polymorphisms with therapeutic efficacy of nateglinide in Chinese patients with T2DM.

\section{Materials and methods Study design and participants}

A total of 78 T2DM patients were recruited for this study according to the inclusion and exclusion criteria, of which 60 (39 men and 21 women) completed the full follow-up and study process. Inclusion and exclusion criteria were developed based on previous studies and T2DM was diagnosed according to the 1999 World Health Organization criteria $[18,22]$. Inclusion criteria were (i) BMI in the range of $18.5-30 \mathrm{~kg} / \mathrm{m}^{2}$ and (ii) patients who were newly diagnosed with T2DM, unmedicated and had

Table 1 Clinical characteristics of T2DM patients before and after nateglinide treatment

\begin{tabular}{lccl}
\hline Parameters & Before treatment & After treatment & P values \\
\hline FPG $(\mathrm{mmol} / \mathrm{L})$ & $8.70 \pm 2.23$ & $6.67 \pm 1.17$ & 0.000 \\
PPG $(\mathrm{mmol} / \mathrm{L})$ & $14.26 \pm 2.94$ & $10.46 \pm 1.59$ & 0.000 \\
FINS $(\mathrm{mU} / \mathrm{L})$ & $9.26 \pm 5.87$ & $14.09 \pm 13.47$ & 0.007 \\
PINS $(\mathrm{mU} / \mathrm{L})$ & $39.33 \pm 33.11$ & $70.21 \pm 52.89$ & 0.000 \\
HOMA-IR & $3.61 \pm 2.42$ & $4.27 \pm 4.28$ & 0.246 \\
HOMA-B & $28.26 \pm 16.01$ & $60.31 \pm 35.32$ & 0.000 \\
HbA1c $(\%)$ & $8.35 \pm 1.74$ & $6.71 \pm 1.00$ & 0.000 \\
TG $(\mathrm{mmol} / \mathrm{L})$ & $2.15 \pm 1.34$ & $1.85 \pm 1.10$ & 0.002 \\
TC $(\mathrm{mmol} / \mathrm{L})$ & $4.98 \pm 1.31$ & $4.58 \pm 1.08$ & 0.001 \\
HDL-C $(\mathrm{mmol} / \mathrm{L})$ & $1.30 \pm 0.42$ & $1.49 \pm 0.68$ & 0.026 \\
LDL-C (mmol/L) & $2.82 \pm 0.78$ & $2.68 \pm 0.74$ & 0.066 \\
\hline
\end{tabular}

Abbreviations: FPG, fasting plasma glucose; PPG, postprandial plasma glucose; FINS, fasting serum insulin; PINS, postprandial serum insulin; HOMA-IR, homeostasis model assessment for insulin resistance; HOMA-B, homeostasis model assessment for beta cell function; $\mathrm{HbA} 1 \mathrm{c}$, hemoglobin $\mathrm{A}_{1 c} ; \mathrm{TG}$, triglyceride; TC, total cholesterol; $\mathrm{HDL}-\mathrm{C}$, high-density lipoprotein-cholesterol; $\mathrm{LDL}-\mathrm{C}$, low-density lipoprotein-cholesterol

Data are expressed as mean \pm SD. $P$ values are determined by the Student's $t$ test 
Table 2 Effects of different PPARD rs2016520 genotypes in T2DM patients on clinical characteristics determined before and after nateglinide treatment

\begin{tabular}{|c|c|c|c|c|}
\hline \multirow[t]{2}{*}{ Parameters } & \multicolumn{3}{|c|}{ PPARD rs2016520 } & \multirow[t]{2}{*}{$P$ values } \\
\hline & & TT & $\mathrm{TC}+\mathrm{CC}$ & \\
\hline N (male/femal) & & $35(24 / 11)$ & $25(15 / 10)$ & $0.493^{b}$ \\
\hline \multirow[t]{3}{*}{$\mathrm{FPG}(\mathrm{mmol} / \mathrm{L})$} & Before & $9.07 \pm 2.63$ & $8.19 \pm 1.42$ & 0.134 \\
\hline & After & $6.74 \pm 1.36$ & $6.57 \pm 0.87222$ & 0.579 \\
\hline & DV & $-2.32 \pm 1.72$ & $-1.61 \pm 1.44$ & 0.099 \\
\hline \multirow[t]{3}{*}{ PPG (mmol/L) } & Before & $15.23 \pm 2.78$ & $12.90 \pm 2.66$ & 0.002 \\
\hline & After & $10.57 \pm 1.71$ & $10.31 \pm 1.42$ & 0.538 \\
\hline & DV & $-4.66 \pm 2.70$ & $-2.59 \pm 2.13$ & 0.002 \\
\hline \multirow[t]{3}{*}{ FINS (mU/L) } & Before & $9.36 \pm 5.93$ & $9.13 \pm 5.91$ & 0.883 \\
\hline & After & $16.06 \pm 15.72$ & $11.32 \pm 9.05$ & 0.182 \\
\hline & DV & $6.70 \pm 15.03$ & $2.19 \pm 10.33$ & 0.201 \\
\hline \multirow[t]{3}{*}{ PINS (mU/L) } & Before & $41.55 \pm 30.72$ & $36.23 \pm 36.61$ & 0.544 \\
\hline & After & $75.14 \pm 54.71$ & $63.29 \pm 50.51$ & 0.397 \\
\hline & DV & $33.59 \pm 43.71$ & $27.07 \pm 29.25$ & 0.519 \\
\hline \multirow[t]{3}{*}{ HOMA-IR } & Before & $3.81 \pm 2.65$ & $2.72 \pm 1.47$ & 0.443 \\
\hline & After & $4.94 \pm 4.94$ & $3.34 \pm 2.97$ & 0.157 \\
\hline & DV & $1.12 \pm 4.85$ & $-0.01 \pm 3.53$ & 0.338 \\
\hline \multirow[t]{3}{*}{ HOMA-B } & Before & $28.25 \pm 16.60$ & $25.22 \pm 14.80$ & 0.469 \\
\hline & After & $73.20 \pm 42.81$ & $50.74 \pm 26.43$ & 0.015 \\
\hline & DV & $44.95 \pm 19.23$ & $25.48 \pm 15.93$ & 0.000 \\
\hline \multirow[t]{3}{*}{$\mathrm{HbA1c}(\%)$} & Before & $8.58 \pm 1.81$ & $7.02 \pm 1.60$ & 0.221 \\
\hline & After & $6.58 \pm 1.12$ & $6.42 \pm 0.80$ & 0.543 \\
\hline & DV & $-1.87 \pm 1.55$ & $-1.60 \pm 1.44$ & 0.714 \\
\hline \multirow[t]{3}{*}{ TG (mmol/L) } & Before & $2.19 \pm 1.58$ & $2.09 \pm 0.92$ & 0.782 \\
\hline & After & $1.90 \pm 1.26$ & $1.78 \pm 0.83$ & 0.690 \\
\hline & DV & $-0.29 \pm 0.78$ & $-0.31 \pm 0.64$ & 0.926 \\
\hline \multirow[t]{3}{*}{ TC (mmol/L) } & Before & $4.67 \pm 1.20$ & $4.45 \pm 0.88$ & 0.906 \\
\hline & After & $4.74 \pm 1.19$ & $4.52 \pm 0.87$ & 0.439 \\
\hline & DV & $-0.29 \pm 0.75$ & $-0.55 \pm 1.12$ & 0.283 \\
\hline \multirow[t]{3}{*}{$\mathrm{HDL}-\mathrm{C}(\mathrm{mmol} / \mathrm{L})$} & Before & $1.29 \pm 0.45$ & $1.31 \pm 0.38$ & 0.788 \\
\hline & After & $1.38 \pm 0.41$ & $1.64 \pm 0.92$ & 0.158 \\
\hline & DV & $0.09 \pm 0.38$ & $0.32 \pm 0.87$ & 0.183 \\
\hline \multirow[t]{3}{*}{ LDL-C (mmol/L) } & Before & $2.79 \pm 0.80$ & $2.87 \pm 0.78$ & 0.720 \\
\hline & After & $2.75 \pm 0.79$ & $2.58 \pm 0.67$ & 0.364 \\
\hline & DV & $-0.04 \pm 0.61$ & $0.29 \pm 0.57$ & 0.110 \\
\hline
\end{tabular}

Abbreviations: FPG, fasting plasma glucose; PPG, postprandial plasma glucose; FINS, fasting serum insulin; PINS, postprandial serum insulin; HOMA-IR, homeostasis model assessment for insulin resistance; HOMA-B, homeostasis model assessment for beta cell function; $\mathrm{HbA1c}$, hemoglobin $\mathrm{A}_{1 \mathrm{c}} ; \mathrm{TG}$, triglyceride; TC, total cholesterol; $\mathrm{HDL}-\mathrm{C}$, high-density lipoprotein-cholesterol; LDL-C, low-density lipoprotein-cholesterol

Data are given as mean \pm SD. $P$ values represent statistical difference between different genotypes assessed by independent-samples t-tests. ${ }^{b} P$ values are determined by the Pearson chi-square test. ${ }^{C} P$ values are determined by the Kruskal-Wallis test

DV, differential values (post-administration minus pre-administration) never use agonists or inhibitors of CYP2C9, CYP3A4 and OATP1B1. Patients on insulin therapy, pregnant or lactating women, and patients with serious diseases such as acute myocardial infarction, cerebrovascular accident, trauma, and severe hepatic and renal disorders were excluded. All subjects who met the enrollment criteria received $360 \mathrm{mg}$ nateglinide per day $(120 \mathrm{mg}$ once before meal) orally for 8 consecutive weeks. The study was registered in the Chinese Clinical Trial Register (No. ChiCTR CCC13003536), in which the protocol used was approved by the ethics committee of the Affiliated Hospital of Xuzhou Medical University. All methods were carried out in accordance with Ethical Review of Drug Clinical Trials in China and followed the Helsinki Declaration II. Written informed consent was obtained from each participant before the study.

\section{Anthropometric and biochemical measurements}

The general anthropometric parameters considered for this study were height (in meters), weight (in kilograms), and waist and hip circumferences (in centimeters). After an overnight fast by the study subjects, blood samples for measurements of plasma glucose and insulin were obtained both in the fasting state and $2 \mathrm{~h}$ later during a standard 75-g oral glucose tolerance test. Plasma glucose and insulin, hemoglobin A1c (HbA1c), and serum lipids were measured as previously described [22]. These parameters were measured at the end of weeks 0 and 8 after administration of nateglinide. The homeostasis model assessment for insulin resistance (HOMA-IR) and beta cell function (HOMA-B) are given by: HOMA$\mathrm{IR}=$ fasting insulin level $(\mathrm{mU} / \mathrm{L}) \times$ fasting glucose level $(\mathrm{mmol} / \mathrm{L}) / 22.5 ;$ HOMA-B $=20 \times$ fasting serum insulin (FINS)/(FPG-3.5) [23].

\section{Genotyping}

Genomic DNA was extracted from peripheral blood leucocytes using a SiMax Genome DNA Kit (Sbsbio, Shanghai, China). In the present study, the PPARD rs2016520 locus was amplified by the polymerase chain reaction (PCR) with the following primers: 5-TGGGAAGGG TGATAGGGCA-3 (forward) and 5'-CTGGTGAGT GGCAGAGCAGA-3 (reverse). The 602 bp PCR products were digested by FoKI (NEB, Beijing, China). For the NOS1AP rs12742393 locus, the following primer pairs were used 5-GGTGAATGTGTACAAAGGAGAAGG-3 (forward) and 5-CAAACTGAAAT GGACCACAAAGA G-3 (reverse). The PCR products were digested by BsrI (NEB, Beijing, China). All obtained DNA fragments were 

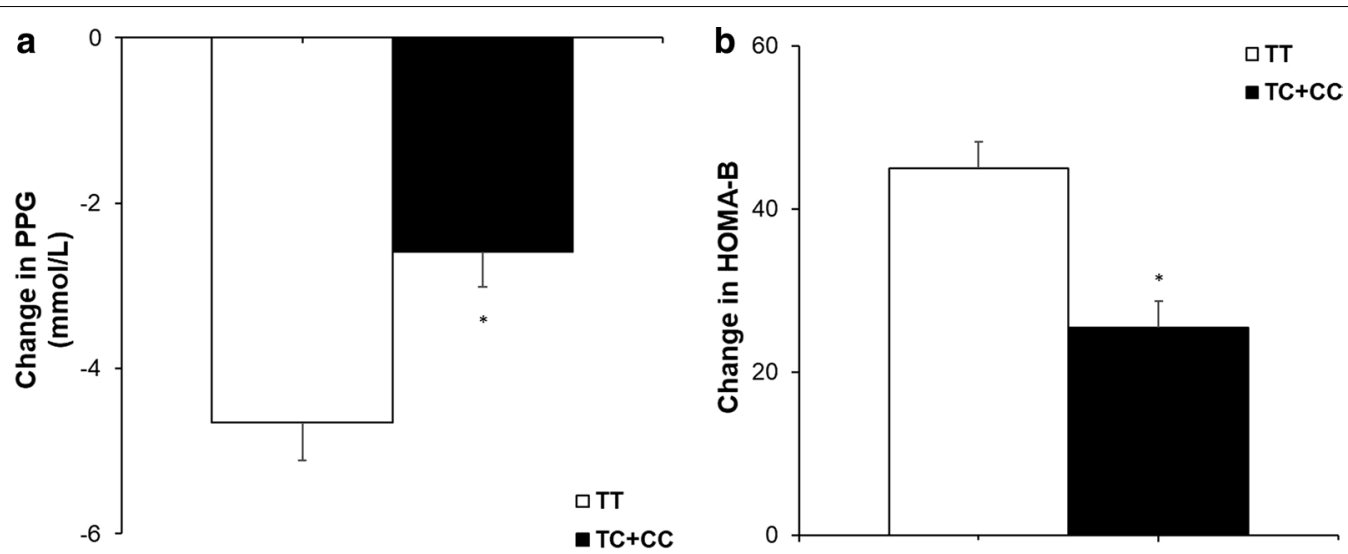

Fig. 1 Comparisons of DV (postadministration minus preadministration) of PPG (a) and HOMA-B (b) between the different PPARD rs2016520 genotypes in T2DM patients after treatment of nateglinide. Data are expressed with mean \pm standard error. ${ }^{*} P<0.05$ compared with TT genotype group

separated by $2 \%$ agarose gel electrophoresis followed by ethidium bromide staining and visualization with UV transillumination. To confirm the assay results, $5.0 \%$ of all samples were directly sequenced.

\section{Definition of the response to nateglinide}

T2DM subjects were classified into two groups based on changes in HbA1c after treatment with nateglinide: responder and non-responder. According to previous studies, nateglinide monotherapy improved HbA1c in patients with T2DM by an average of $10 \%$ to $20 \%$ from baseline levels [24-26]. In the present study, HbA1c levels were reduced by an average of $19.95 \%$ in all subjects treated with nateglinide. Therefore, we identified a $20 \%$ improvement in HbA1c after 8 weeks of nateglinide treatment as an intermediate value, with responders were defined as patients with $20 \%$ or greater decrease in $\mathrm{HbA} 1 \mathrm{c}$ and non-responders defined as patients who failed to achieve this level.

\section{Statistical analysis}

Statistical analyses were carried out using SPSS software (version 16.0, SPSS Inc., Chicago, IL). The measurement data and count data were expressed as mean \pm standard deviation (SD) and proportion, respectively. Frequencies of genotypes were assessed using $X^{2}$ tests in the study sample. Parameters before and after treatment were compared by paired $t$-test. Independent samples $t$ tests were used to estimate the effects of nateglinide on biochemical index among genotypes [22]. Parameters with nonnormal distribution were analyzed by the Kruskal-Wallis test. $\alpha=0.05$ was used as the test level.

\section{Results}

Clinical efficacy evaluation of nateglinide

To evaluate the effects of PPARD and NOS1AP variations on the efficacy of nateglinide, 60 newly diagnosed T2DM patients with various PPARD rs2016520 (C/T) and NOS1AP rs12742393 (A/C) genotypes but with the same SLCO1B1 T521C and CYP2C9*1 genotype were enrolled. Nateglinide significantly decreased the levels of FPG, PPG, HbA1c, TG, and TC, and increased the levels of FINS, PINS, HOMA-B and HDL-C levels in patients with T2DM after 8 weeks of nateglinide treatment (Table 1).

\section{Effects of the rs2016520 and rs12742393 polymorphisms on therapeutic efficacy of nateglinide in patients with T2DM}

Patients with PPARD rs2016520 TT genotypes had a significantly decrease in PPG and notably increase HOMA$\mathrm{B}$ as compared with patients with the TC $+\mathrm{CC}$ genotypes, which indicated that patients with genotype TT had better efficacy of nateglinide monotherapy (Table 2, Fig. 1). Moreover, patients with NOS1AP rs12742393 AC + CC genotypes had poor response of nateglinide with respect to FPG, FINS, HOMA-IR, and HOMA-B compared with AA genotype carriers (Table 3, Fig. 2).

Association of PPARD rs2016520 and NOS1AP rs12742393 genetic polymorphisms with response rate to nateglinide treatment

In order to evaluate the association of PPARD and NOS1AP polymorphisms with the response to nateglinide treatment, genotypes and allelic frequency distributions were analyzed in the responder and non-responder 
Table 3 Comparisons of clinical characteristics in T2DM patients with different NOS1AP rs12742393 genotypes before and after nateglinide treatment

\begin{tabular}{|c|c|c|c|c|}
\hline Parameters & & AA & $\mathrm{AC}+\mathrm{CC}$ & $P$ value \\
\hline N(male/female) & & $24(17 / 7)$ & $36(22 / 14)$ & $0.598^{b}$ \\
\hline \multirow[t]{3}{*}{ FPG (mmol/L) } & Before & $9.97 \pm 2.54$ & $10.32 \pm 2.02$ & 0.580 \\
\hline & After & $6.51 \pm 1.34$ & $8.75 \pm 1.44$ & 0.000 \\
\hline & DV & $-3.48 \pm 2.55$ & $-1.57 \pm 1.28$ & $0.000^{c}$ \\
\hline \multirow[t]{3}{*}{ PPG (mmol/L) } & Before & $17.25 \pm 4.31$ & $16.73 \pm 4.50$ & 0.651 \\
\hline & After & $10.47 \pm 3.45$ & $12.95 \pm 3.68$ & 0.016 \\
\hline & DV & $-6.78 \pm 4.41$ & $-4.81 \pm 3.57$ & $0.000^{c}$ \\
\hline \multirow[t]{3}{*}{ FINS (mU/L) } & Before & $9.33 \pm 6.49$ & $8.92 \pm 5.96$ & 0.802 \\
\hline & After & $10.04 \pm 6.26$ & $12.87 \pm 6.81$ & 0.110 \\
\hline & DV & $0.72 \pm 5.15$ & $3.98 \pm 4.65$ & 0.014 \\
\hline \multirow[t]{3}{*}{ PINS (mU/L) } & Before & $31.63 \pm 22.32$ & $31.90 \pm 21.62$ & 0.963 \\
\hline & After & $46.91 \pm 26.82$ & $47.82 \pm 26.90$ & 0.028 \\
\hline & DV & $14.31 \pm 14.23$ & $17.36 \pm 15.33$ & 0.441 \\
\hline \multirow[t]{3}{*}{ HOMA-IR } & Before & $4.04 \pm 2.96$ & $4.02 \pm 2.58$ & 0.978 \\
\hline & After & $2.81 \pm 1.66$ & $4.32 \pm 2.21$ & 0.006 \\
\hline & DV & $-1.22 \pm 2.07$ & $0.21 \pm 1.23$ & 0.001 \\
\hline \multirow[t]{3}{*}{ HOMA-B } & Before & $25.45 \pm 17.21$ & $27.01 \pm 16.92$ & 0.730 \\
\hline & After & $75.20 \pm 43.81$ & $47.7 \pm 39.31$ & 0.014 \\
\hline & DV & $45.95 \pm 37.23$ & $22.48 \pm 21.93$ & 0.003 \\
\hline \multirow[t]{3}{*}{$\mathrm{HbA1c}(\%)$} & Before & $9.81 \pm 1.89$ & $9.68 \pm 1.96$ & 0.809 \\
\hline & After & $7.02 \pm 0.78$ & $7.01 \pm 1.74$ & 0.979 \\
\hline & DV & $-2.79 \pm 1.58$ & $-2.71 \pm 1.28$ & 0.830 \\
\hline \multirow[t]{3}{*}{$\mathrm{TG}(\mathrm{mmol} / \mathrm{L})$} & Before & $2.21 \pm 1.53$ & $2.51 \pm 2.26$ & 0.572 \\
\hline & After & $1.84 \pm 1.04$ & $2.06 \pm 2.02$ & 0.625 \\
\hline & DV & $-0.36 \pm 1.13$ & $-0.37 \pm 2.04$ & 0.983 \\
\hline \multirow[t]{3}{*}{ TC (mmol/L) } & Before & $5.10 \pm 1.01$ & $5.32 \pm 1.78$ & 0.585 \\
\hline & After & $5.04 \pm 0.91$ & $4.74 \pm 1.23$ & 0.311 \\
\hline & DV & $-0.06 \pm 0.91$ & $-0.54 \pm 1.47$ & 0.806 \\
\hline \multirow[t]{3}{*}{$\mathrm{HDL}-\mathrm{C}(\mathrm{mmol} / \mathrm{L})$} & Before & $1.41 \pm 0.42$ & $1.39 \pm 0.49$ & 0.871 \\
\hline & After & $1.37 \pm 0.39$ & $1.30 \pm 0.43$ & 0.524 \\
\hline & DV & $-0.06 \pm 0.45$ & $-0.11 \pm 0.66$ & 0.747 \\
\hline \multirow[t]{3}{*}{ LDL-C (mmol/L) } & Before & $3.10 \pm 0.82$ & $3.19 \pm 1.16$ & 0.744 \\
\hline & After & $3.31 \pm 0.91$ & $3.02 \pm 1.12$ & 0.295 \\
\hline & DV & $0.21 \pm 0.92$ & $-0.03 \pm 1.37$ & 0.455 \\
\hline
\end{tabular}

Abbreviations: FPG, fasting plasma glucose; PPG, postprandial plasma glucose; FINS, fasting serum insulin; PINS, postprandial serum insulin; HOMA-IR, homeostasis model assessment for insulin resistance; $H O M A-B$, homeostasis model assessment for beta cell function; $\mathrm{HbA1c}$, hemoglobin $\mathrm{A}_{1 \mathrm{c}} ; \mathrm{TG}$, triglyceride; TC, total cholesterol; $\mathrm{HDL}-\mathrm{C}$, high-density lipoprotein-cholesterol; LDL-C, low-density lipoprotein-cholesterol

Data are given as mean \pm standard deviation. $P$ values represent statistical difference between different genotypes assessed by independent-samples t-tests. ${ }^{b} P$ values are determined by Pearson chi-square test. ${ }^{c} P$ values are determined by Kruskal-Wallis test

DV, differential values (postadministration minus preadministration)

groups (Table 4). No signifficant effects of the variation in PPARD rs2016520 $(\mathrm{T} / \mathrm{C})$ on nateglinide treatment were detected. According to predetermined criteria of $20 \%$ reduction from baseline, NOS1AP rs12742393 A allele carriers exhibited higher response rate to nateglinide treatment; AA allele homozygotes had the highest response rate $(70.83 \%)$, while $\mathrm{AC}$ heterozygous and $\mathrm{CC}$ homozygous had $44.44 \%$ and $22.22 \%$, respectively $(P=0.006)$.

\section{Discussion}

In the present study, we found for the first time that genetic polymorphisms of PPARD and NOS1AP may affect the therapeutic efficacy of nateglinide in Chinese patients with T2DM. We observed that, T2DM patients with at least one $C$ allele of PPARD rs2016520 (T/C) or one C allele of NOS1AP rs12742393 (A/C) may be less responsive to treatment with nateglinide, indicating that the PPARD and NOS1AP genotype may serve as nateglinide response prognosticator. Therefore, we suggest that prior genotyping and individualized administration of nateglinide may be beneficial for those T2DM patients who require treatment with nateglinide. PPARD and NOS1AP are directly or indirectly involved in the regulation of $\beta$-cell function and insulin resistance, which suggests that genetic polymorphisms in the two genes may contribute to interindividual differences in nateglinide response [7-9, 16, 17]. Further pharmacogenetic and functional studies are necessary to investigate the potential mechanism and lay the foundation for Individualized administration for patients with T2DM.

Studies have reported that CYP2C9 and SLCO1B1 gene polymorphisms could affect the pharmacokinetic process of nateglinide, resulting in differences in drug concentrations in plasma and therapeutic efficacy [27-30]. Therefore, we selected patients with the same CYP2C9*1 and SLCO1B1 521TT genotype to avoid any possible changes in the pharmacokinetics and pharmacodynamics of nateglinide caused by OATP1B1 or CYP2C9 polymorphism. After the treatment with nateglinide for 8 consecutive weeks, serum FPG, PPG, HbA1c, TG and TC levels decreased significantly in patients with T2DM and FINS, PINS, HOMA-B and HDL-C levels increased markedly increased markedly. Also, patients with PPARD rs2016520 TC + CC genotypes had attenuated efficacy of nateglinide monotherapy with respect to PPG and HOMA-B compared with TT genotype carriers. Our results also showed that the NOS1AP rs12742393 (A/C) polymorphism was associated with an attenuated nateglinide effect in Chinese patients with T2DM, and that individuals with $\mathrm{AC}+\mathrm{CC}$ genotypes showed a smaller increase in FINS and HOMA-B, but a smaller decrease in FPG and HOMA-IR levels as compared to individuals with the TT genotype.

PPARD encoding PPAR- $\delta$, which is related to islet function and insulin resistance, might directly or indirectly 

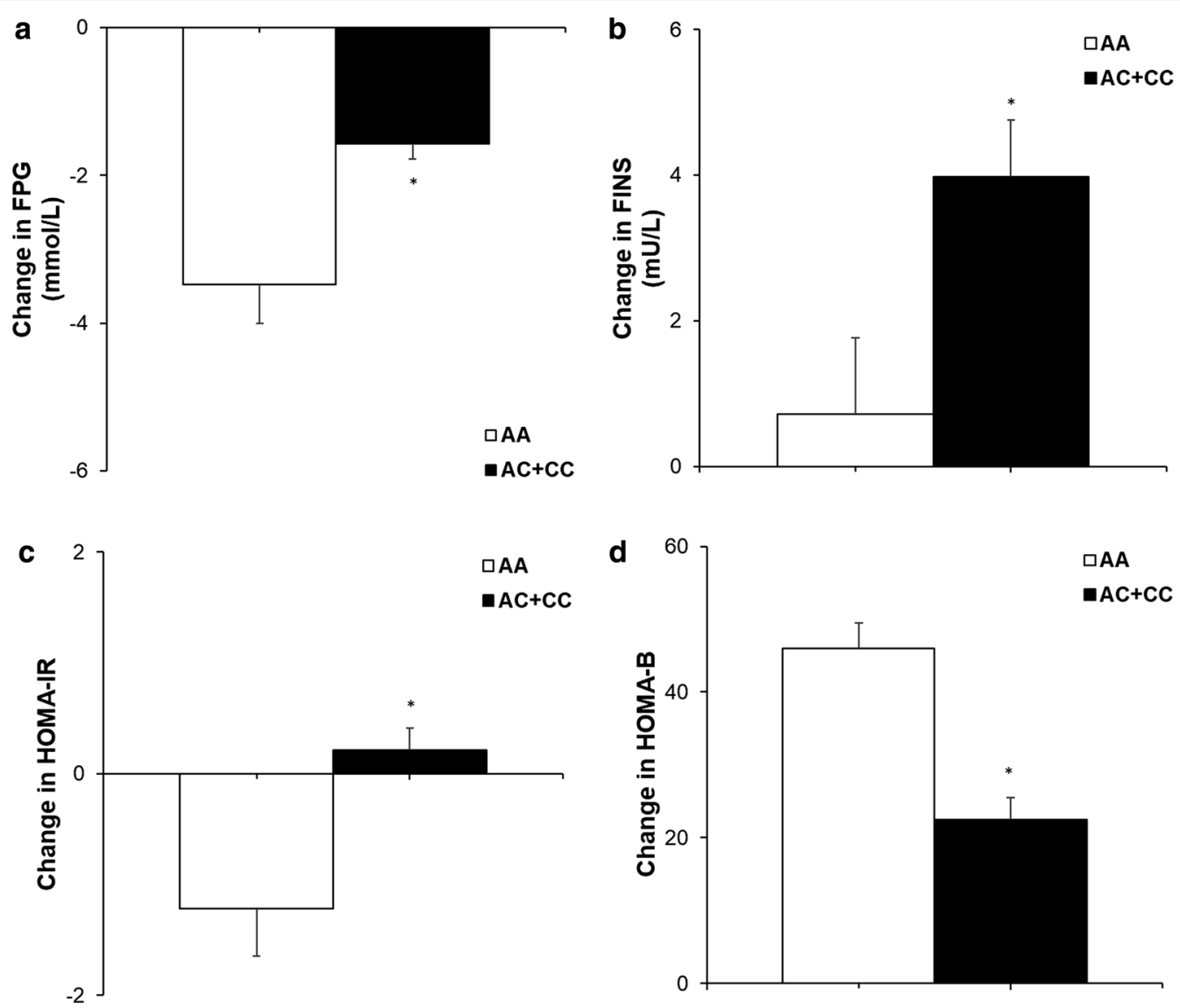

Fig. 2 Comparisons of DV (postadministration minus preadministration) of FPG (a), FINS (b), HOMA-IR (c) and HOMA-B (d) among the different NOS1AP rs 12742393 genotypes in T2DM patients after treatment of nateglinide. Data are expressed with mean \pm standard error. ${ }^{*} P<0.05$ compared with AA genotype group

Table 4 Genotype and allele distributions between responders and non-responders of PPARD rs2016520 and NOS1AP rs12742393 variants $(n=60)$

\begin{tabular}{|c|c|c|c|c|c|c|c|}
\hline \multirow[t]{2}{*}{ PPARD rs2016520 } & \multicolumn{3}{|l|}{ Genotype } & \multirow[t]{2}{*}{$P$ value } & \multicolumn{2}{|c|}{ Allele frequency } & \multirow[t]{2}{*}{$P$ value } \\
\hline & TT & TC & $\mathrm{CC}$ & & $T$ & $\mathrm{C}$ & \\
\hline Responder (\%) & $19(54.29 \%)$ & $11(47.83 \%)$ & $1(50.00 \%)$ & & $49(52.69 \%)$ & $13(48.15 \%)$ & \\
\hline Non-responder (\%) & $16(45.71 \%)$ & $12(52.17 \%)$ & $1(50.00 \%)$ & 0.964 & $44(47.31 \%)$ & $14(51.85 \%)$ & 0.678 \\
\hline NOS1AP rs 12742393 & $\mathrm{AA}$ & $A C$ & CC & & A & $\mathrm{C}$ & \\
\hline Responder (\%) & 17 (70.83\%) & $12(44.44 \%)$ & $2(22.22 \%)$ & & $46(61.33 \%)$ & $16(35.56 \%)$ & \\
\hline Non-responder (\%) & $7(29.17 \%)$ & $15(55.56 \%)$ & $7(77.78 \%)$ & 0.027 & $29(38.67 \%)$ & $29(64.44 \%)$ & 0.006 \\
\hline
\end{tabular}

participate in the pathogenesis of T2DM [9, 31-33]. In the present study, we preliminarily found that the polymorphism of PPARD affected the impact of nateglinide on insulin secretion in Chinese patients with T2DM, which may be attributed entirely to the role of PPAR- $\delta$ in insulin secretion, as measured by HOMA-B. The biological effect of PPAR- $\delta$ overlaps with the therapeutic mechanism of nateglinide to a certain extent, which can partly explain the mechanism of PPARD genetic polymorphism affecting the efficacy of nateglinide. However, the exact molecular mechanism remains to be further studied.

NOS1AP mainly regulates nNOS activity, and nNOS can inhibit intracellular $\mathrm{Ca}^{2+}$ level and thereby regulate insulin secretion $[15,18,34]$. In addition, lateral ventricular injection of nNOS inhibitors can affect insulin secretion and insulin sensitivity [15]. In this study, we 
observed that subjects with at least one $\mathrm{C}$ allele of the NOS1AP rs12742393 showed a smaller decrease in FPG and HOMA-IR and more obvious increase in FINS and HOMA-B levels than those with the AA genotype, which suggested that the NOS1AP rs12742393 C allele confers the poor nateglinide response through improving insulin resistance, as measured by HOMA-IR. Animal studies have shown that knockout of mouse nNOS gene may induce insulin resistance in mice [35]. Meanwhile, it has been reported that the dysfunction of nNOS in islet $\beta$ cells is related to insulin secretion $[15,16]$. Therefore, it is speculated that NOS1AP rs12742393 risk gene C affects the efficacy of nateglinide in patients with T2DM, which is at least partially associated with insulin resistance and islet $\beta$ cell. However, the exact mechanism by which NOS1AP polymorphism affects the efficacy of nateglinide needs to be further investigated.

In interpreting the results of our study, several shortcomings must be addressed. First, our study focused only on the effect of variation in PPARD rs2016520 and NOS1AP rs12742393 on nateglinide efficacy. However, the possibility still exists that other susceptibility loci for T2DM may affect the therapeutic efficacy of nateglinide. Second, the sample size was relatively small, we may have missed some meaningful results. The duration of nateglinide therapy for 8 weeks is relatively short, since HbA1c reduction is better observed after 12 weeks of treatment. Further studies with a larger sample size and longer observation periods are required to confirm the effects of PPARD and NOS1AP polymorphisms on the therapeutic efficacy of nateglinide. Third, our study only investigated the effects of gene polymorphism on the efficacy of nateglinide. However, the mechanisms by which the two SNPs in PPARD and NOS1AP affect the therapeutic efficacy of nateglinide are not fully understood. In the future, more functional studies are needed to explore the mechanism by which PPARD and NOS1AP genetic polymorphisms affect drug efficacy.

\section{Conclusion}

The efficacy profile of primary diabetic patients receiving nateglinide monotherapy was associated with PPARD rs2016520 and NOS1AP rs12742393 polymorphisms. Prior genetic analysis of PPARD rs2016520 (T/C) and NOS1AP rs12742393 (A/C) is a useful idea worthy of further exploration to achieve individualized drug delivery.

\footnotetext{
Abbreviations

T2DM: Type 2 diabetes mellitus; FPG: Fasting plasma glucose; PPG: Post plasma glucose; BMI: Body mass index; PCR-RFLP: Polymerase chain reaction-restriction fragment length polymorphism; TG: Triglycerides; TC: Total cholesterol; LDL-c: Low-density cholesterol; HDL-c: High-density cholesterol; HbA1c: Glycated hemoglobin; HOMA-IR: Homeostasis model assessment for insulin
}

resistance; HOMA- $\beta$ : Homeostasis model assessment for islet $\beta$ cell function; FINS: Fasting serum insulin; PINS: Postprandial serum insulin.

\section{Acknowledgements}

We thank all the volunteers in this study for their cooperation, and the physicians from the Department of Endocrinology, the Affiliated Hospital of Xuzhou Medical University for their support.

\section{Authors' contributions}

All authors contributed substantially to the work presented in this paper, read and approved the final manuscript. TW and J-FS designed all the work under the supervision of QL and X-XY. QL designed the research, contributed substantially with data analysis, results interpretations and manuscript editing and approval. X-YZ and C-LL collected the patients' data and did indicators analysis. All authors read and approved the final manuscript.

\section{Funding}

This work was supported by Wuxi Science and Technology Development Medical and Health Guidance Project (No. CSZON1809) and Jiangsu Research Hospital Association for Precision Medication (No. JY202011). The authors declare that they have no financial relationship with the organization that sponsored the research, and the funding body was not involved in study design, data collection, analysis and writing of the study.

\section{Availability of data and materials}

The datasets generated and analyzed during the current study are available in the link"https://submit.ncbi.nlm.nih.gov/subs/variation_file/SUB9598166/ overview". The accession number is SUB9598166.

\section{Declarations}

\section{Ethics approval and consent to participate}

Manuscript reporting studies involving human participants, human data or human tissue are reported with approved statement on Ethical Review of Drug Clinical Trials in China and follow the Helsinki Declaration II. Written informed consent was obtained from each participant before the study.

\section{Consent for publication}

Not applicable.

\section{Competing interests}

The authors declare that they have no conflicts of interest.

\section{Author details}

${ }^{1}$ Jiangsu Key Laboratory of New Drug Research and Clinical Pharmacy, Xuzhou Medical University, Xuzhou, China. ${ }^{2}$ Department of Pharmacy, Affiliated Hospital of Xuzhou Medical University, Xuzhou, China. ${ }^{3}$ Department of Pharmacy, Affiliated Hospital of Jiangnan University, Wuxi, China.

Received: 14 July 2021 Accepted: 18 October 2021

Published online: 12 November 2021

\section{References}

1. Cai $X L$, Luo YY, Han XY, Ji LN. A meta-analysis of efficacy and safety of nateglinide in type 2 diabetes mellitus in Asia. Chin J Diabetes. 2012;21:9137. https://doi.org/10.3969/j.issn.1006-6187.2013.10.014.

2. Cheng Y, Xiong QX, Liu Q, et al. A comparative study of the clinical efficacy of naglinide and acarbose in the treatment of type 2 diabetes mellitus. Modern Chin Drug Use. 2010;4:166-7. https://doi.org/10.3969/j. issn.1673-9523.2010.12.144.

3. Zhou S, Xiang Q, Mu G, et al. Effects of CYP2C8 and SLCO1B1 genetic polymorphisms on repaglinide pharmacokinetics: a systematic review and meta-analysis. Curr Drug Metab. 2019;20(4):266-74. https://doi.org/ 10.2174/1389200220666190111114146.

4. Barroso I, Luan J, Middelberg RP, et al. Candidate gene association study in type 2 diabetes indicates a role for genes involved in beta-cell function as well as insulin action [published correction appears in Plos Biol. 
2003 ;1(3):445]. PLoS Biol. 2003;1 (1):E20. https://doi.org/10.1371/journal. pbio.0000020.

5. Saad MAE, Fahmy MIM, Al-Shorbagy M, et al. Nateglinide exerts neuroprotective effects via downregulation of HIF-1 a/TIM-3 inflammatory pathway and promotion of caveolin-1 expression in the rat's hippocampus subjected to focal cerebral ischemia/reperfusion injury. Inflammation. 2020;43(2):401-16. https://doi.org/10.1007/ s10753-019-01154-3.

6. Wagner N, Wagner KD. PPAR beta/delta and the hallmarks of cancer. Cells. 2020;9(5):1133. https://doi.org/10.3390/cells9051133.

7. Cao $M$, Tong $Y, L v Q$, et al. PPAR $\delta$ activation rescues pancreatic $\beta$-cell line INS-1E from palmitate-induced endoplasmic reticulum stress through enhanced fatty acid oxidation. PPAR Res. 2012;2012: 680684 https://doi.org/10.1155/2012/680684.

8. Iglesias J, Barg S, Vallois D, et al. PPAR $\beta / \delta$ affects pancreatic $\beta$ cell mass and insulin secretion in mice. J Clin Investig. 2012;122(11):4105-17. https://doi.org/10.1172/JCl42127.

9. Han L, Shen WJ, Bittner S, et al. PPARs: regulators of metabolism and as therapeutic targets in cardiovascular disease. Part II: PPAR- $\beta / \delta$ and PPAR- $\gamma$. Future Cardiol. 2017;13(3):279-96. https://doi.org/10.2217/fca-2017-0019.

10. Bojic $L A$, Telford $D E$, Fullerton $M D$, et al. PPAR $\delta$ activation attenuates hepatic steatosis in Ldlr-/- mice by enhanced fat oxidation, reduced lipogenesis, and improved insulin sensitivity. J Lipid Res. 2014;55(7):125466. https://doi.org/10.1194/jlr.M046037.

11. Greene NP, Fluckey JD, Lambert BS, et al. Regulators of blood lipids and lipoproteins? PPARS and AMPK, induced by exercise, are correlated with lipids and lipoproteins in overweight/obese men and women. Am J Physiol Endocrinol Metab. 2012;303(10):E1212-21. https://doi.org/10. 1152/ajpendo.00309.2012.

12. Tang L, Lü Q, Cao H, et al. PPARD rs2016520 polymorphism is associated with metabolic traits in a large population of Chinese adults. Gene. 2016;585(2):191-5. https://doi.org/10.1016/j.gene.2016.02.035.

13. Hu C, Jia W, Fang Q, et al. Peroxisome proliferator-activated receptor (PPAR) delta genetic polymorphism and its association with insulin resistance index and fasting plasma glucose concentrations in Chinese subjects. Diabetes Med. 2006;23(12):1307-12. https://doi.org/10.1111/j. 1464-5491.2006.02001.x.

14. Carrillo-Venzor MA, Erives-Anchondo NR, Moreno-González JG, et al. Pro12Ala PPAR- $\gamma 2$ and +294T/C PPAR- $\delta$ polymorphisms and association with metabolic traits in teenagers from Northern Mexico. Genes (Basel). 2020;11(7):776. https://doi.org/10.3390/genes11070776.

15. Gheibi S, Ghasemi A. Insulin secretion: the nitric oxide controversy. EXCLI J. 2020;19:1227-45. https://doi.org/10.17179/excli2020-2711.

16. Shankar RR, Wu Y, Shen HQ, et al. Mice with gene disruption of both endothelial and neuronal nitric oxide synthase exhibit insulin resistance. Diabetes. 2000;49(5):684-7. https://doi.org/10.2337/diabetes.49.5.684.

17. Hao M, Head WS, Gunawardana SC, et al. Direct effect of cholesterol on insulin secretion: a novel mechanism for pancreatic beta-cell dysfunction. Diabetes. 2007;56(9):2328-38. https://doi.org/10.2337/db07-0056.

18. Becker ML, Aarnoudse AJ, Newton-Cheh C, et al. Common variation in the NOS1AP gene is associated with reduced glucose-lowering effect and with increased mortality in users of sulfonylurea. Pharmacogenet Genom. 2008;18(7):591-7. https://doi.org/10.1097/FPC.0b013e3283 $00 \mathrm{e} 8 \mathrm{c} 5$.

19. Becker ML, Visser LE, Newton-Cheh C, et al. Genetic variation in the NOS1 AP gene is associated with the incidence of diabetes mellitus in users of calcium channel blockers. Diabetologia. 2008;51(11):2138-40. https://doi.org/10.1007/s00125-008-1143-4.

20. Hu C, Wang C, Zhang R, et al. Association of genetic variants of NOS1AP with type 2 diabetes in a Chinese population. Diabetologia. 2010;53(2):290-8. https://doi.org/10.1007/s00125-009-1594-2.
21. Wratten NS, Memoli H, Huang Y, et al. Identification of a schizophreniaassociated functional noncoding variant in NOS1 AP [published correction appears in Am J Psychiatry. 2010 Jul;167(7):870]. Am J Psychiatry. 2009;166(4):434-41. https://doi.org/10.1176/appi.ajp.2008.08081266.

22. Wang T, Wang XT, Lai R, et al. MTNR1B gene polymorphisms are associated with the therapeutic responses to repaglinide in Chinese patients with type 2 diabetes mellitus. Front Pharmacol. 2019;10:1318. https://doi. org/10.3389/fphar.2019.01318.

23. Kong $X$, Xing $X$, Hong J, et al. Association of a type 2 diabetes genetic risk score with insulin secretion modulated by insulin sensitivity among Chinese Hans. Clin Genet. 2017;91(6):832-42. https://doi.org/10.1111/ cge.12817.

24. Bolen $\mathrm{S}$, Wilson L, Vassy J, et al. Comparative effectiveness and safety of oral diabetes medications for adults with type 2 diabetes. Rockville, MD: Agency for Healthcare Research and Quality (US); 2007.

25. Kawamori R, Kaku K, Hanafusa T, et al. Efficacy and safety of repaglinide vs nateglinide for treatment of Japanese patients with type 2 diabetes mellitus. J Diabetes Investig. 2012;3(3):302-8. https://doi.org/10.1111/j. 2040-1124.2011.00188.x.

26. Kim MK, Suk JH, Kwon MJ, et al. Nateglinide and acarbose for postprandial glucose control after optimizing fasting glucose with insulin glargine in patients with type 2 diabetes. Diabetes Res Clin Pract. 2011;92(3):3228. https://doi.org/10.1016/j.diabres.2011.01.022.

27. Mannino GC, Andreozzi F, Sesti G. Pharmacogenetics of type 2 diabetes mellitus, the route toward tailored medicine. Diabetes Metab Res Rev. 2019;35(3): e3109. https://doi.org/10.1002/dmrr.3109.

28. Cheng Y, Wang G, Zhang W, et al. Effect of CYP2C9 and SLCO1B1 polymorphisms on the pharmacokinetics and pharmacodynamics of nateglinide in healthy Chinese male volunteers. Eur J Clin Pharmacol. 2013;69(3):407-13. https://doi.org/10.1007/s00228-012-1364-9.

29. Niemi M, Backman JT, Kajosaari LI, et al. Polymorphic organic anion transporting polypeptide 1B1 is a major determinant of repaglinide pharmacokinetics. Clin Pharmacol Ther. 2005;77(6):468-78. https://doi.org/10. 1016/j.clpt.2005.01.018

30. Izumi S, Nozaki Y, Maeda K, et al. Investigation of the impact of substrate selection on in vitro organic anion transporting polypeptide 1B1 inhibition profiles for the prediction of drug-drug interactions. Drug Metab Dispos. 2015:43(2):235-47. https://doi.org/10.1124/dmd.114.059105.

31. Takada I, Makishima M. Peroxisome proliferator-activated receptor agonists and antagonists: a patent review (2014-present). Expert Opin Ther Pat. 2020;30(1):1-13. https://doi.org/10.1080/13543776.2020.1703952.

32. Reilly $\mathrm{SM}$, Lee $\mathrm{CH}$. PPAR delta as a therapeutic target in metabolic disease. FEBS Lett. 2008;582(1):26-31. https://doi.org/10.1016/j.febslet.2007.11. 040.

33. Winzell MS, Wulff EM, Olsen GS, et al. Improved insulin sensitivity and islet function after PPARdelta activation in diabetic $\mathrm{db} / \mathrm{db}$ mice. Eur J Pharmacol. 2010;626(2-3):297-305. https://doi.org/10.1016/j.ejphar.2009.09.053.

34. Icli B, Wu W, Ozdemir D, et al. MicroRNA-615-5p regulates angiogenesis and tissue repair by targeting AKT/eNOS (protein kinase B/endothelial nitric oxide synthase) signaling in endothelial cells. Arterioscler Thromb Vasc Biol. 2019;39(7):1458-74. https://doi.org/10.1161/ATVBAHA.119. 312726.

35. Turini $P$, Thalmann $S$, Jayet $P Y$, et al. Insulin resistance in mice lacking neuronal nitric oxide synthase is related to an alpha-adrenergic mechanism. Swiss Med Wkly. 2007;137(49-50):700-4.

\section{Publisher's Note}

Springer Nature remains neutral with regard to jurisdictional claims in published maps and institutional affiliations. 